\title{
Study of Mn Content Inversion Based on Hyperspectral
}

\author{
Jinbao Liu' ${ }^{1,2,3, ~ *, ~ L e i ~ S h i ~}{ }^{1,2,3}$ \\ ${ }^{1}$ Institute of Land Engineering and Technology, Shaanxi Provincial Land Engineering Construction Group, \\ Xi'an 710075, China \\ ${ }^{2}$ Key Laboratory of Degraded and Unused Land Consolidation Engineering, the Ministry of Land and \\ Resources, Xi'an 710075, China \\ ${ }^{3}$ Shaanxi Provincial Land Engineering Construction Group, Xi'an 710075, China \\ Corresponding author e-mail: liujinbao1212@163.com
}

Keywords: Hyperspectral, Mn Content Inversion, soil samples

\begin{abstract}
In this study, the contents of $\mathrm{Mn}$ in 44 soil samples were collected from Shaanxi Province and were used ASD FieldSpec HR (350 $2500 \mathrm{~nm})$, then the NOR, MSC and SNV of the reflectance were pretreated, and the different deviation and reflectance reciprocal logarithmic transformation were carried out. The optimal hyperspectral estimation model of nine heavy metal elements of Mn was established by regression method, comparing the reflection characteristics of different heavy metal contents and the effect of different pretreatment methods on the establishment of soil heavy metal spectral inversion model. The results show that: (1) The reflectance spectrum improves the signal-to-noise ratio of the reflectance spectrum after the transformation of NOR, MSC and SNV. Combining differential transformation can improve the information of heavy metal elements in the soil, and using the correlation band energy can significantly improve the stability and predictability of the model. (2) The modeling accuracy of the optimal model of Mn spectra of Mn by PLSR method were 0.7852 .
\end{abstract}

\section{Introduction}

In recent years, domestic and foreign scholars have made extensive research on the use of hyperspectral inversion of soil organic matter, nitrogen, phosphorus and potassium (WU and WU., et al., 2005; Han and Liu, et al.,2017; Liu and Mao et al., 2015; Lu and Yao et al., 2015). Used the partial least squares regression model to estimate the cadmium and zinc contents in the Rhine basin (Kooistral and Wehrens et al., 2001). Established a prediction model for the near-infrared and mid-infrared diffuse reflectance spectra in the soils of iron, cadmium, copper, lead, nickel and zinc in the soil of Tarnowskie Gory, Poland (Grzegorz and Gregory et al., 2004).

In this study, nine kinds of heavy metal elements in Shaanxi Province were used as target attributes, and normalization (NOR), Multiplicative Scatter Correction (MSC), standard normal variable transformation (SYV) was reconstructed by Standard Normal Variate (SNV). Combining with the Savitzky - Golay convolution smoothing method, the spectral curves were smoothed and denoised, and the first deviation, second deviation and reciprocal logarithmic differential transformation were combined.

\section{Materials and Methods}

In this study, the main soil types were soil in Shaanxi Province. The soil samples were collected according to the "S" -shaped sampling method. The sampling depth was the thickness of the tillage layer, usually $0-30 \mathrm{~cm}$. A total of 44 soil samples were sampled. The samples were air-dried and passed through the $2 \mathrm{~mm}$ hole. A $200 \mathrm{~g}$ soil sample was mixed and passed through a 100 mesh sieve for indoor heavy metal content and another soil sample was used for soil reflectance spectroscopy. The statistical characteristics of nine heavy metal elements were shown in Table 1. 
Table 1. Statistical result of heavy metal elements for soil samples

\begin{tabular}{ccccccc}
\hline Elements & Samples & Maximum(mg/kg) & Minimum(mg/kg) & Mean(mg/kg) & $\begin{array}{c}\text { Standard } \\
\text { deviation(mg/kg) }\end{array}$ & $\begin{array}{c}\text { Coefficient } \\
\text { of variation }\end{array}$ \\
\hline Mn & 44 & 310 & 249 & 274 & 13.5983 & 0.049628933 \\
\hline
\end{tabular}

\subsection{Data and pre-processing}

The reflectance of 44 samples of soil samples was measured using the ASD FieldSpec4 Spectrometer. The wavelength range of the spectrometer was $350 \sim 2500 \mathrm{~nm}$, the sampling band width was $1.3 \mathrm{~nm}(350 \sim 1000 \mathrm{~nm})$ and $2 \mathrm{~nm}(1000 \sim 2500 \mathrm{~nm})$, and the sampling intervalwas1 nm, totaling 2150 bands. Due to the difference in the energy response of the spectrometer, there was a breakpoint at $1000 \mathrm{~nm}$, and the spectral curve was used for breakpoint repair using Splice Correlation (Fig. 1).

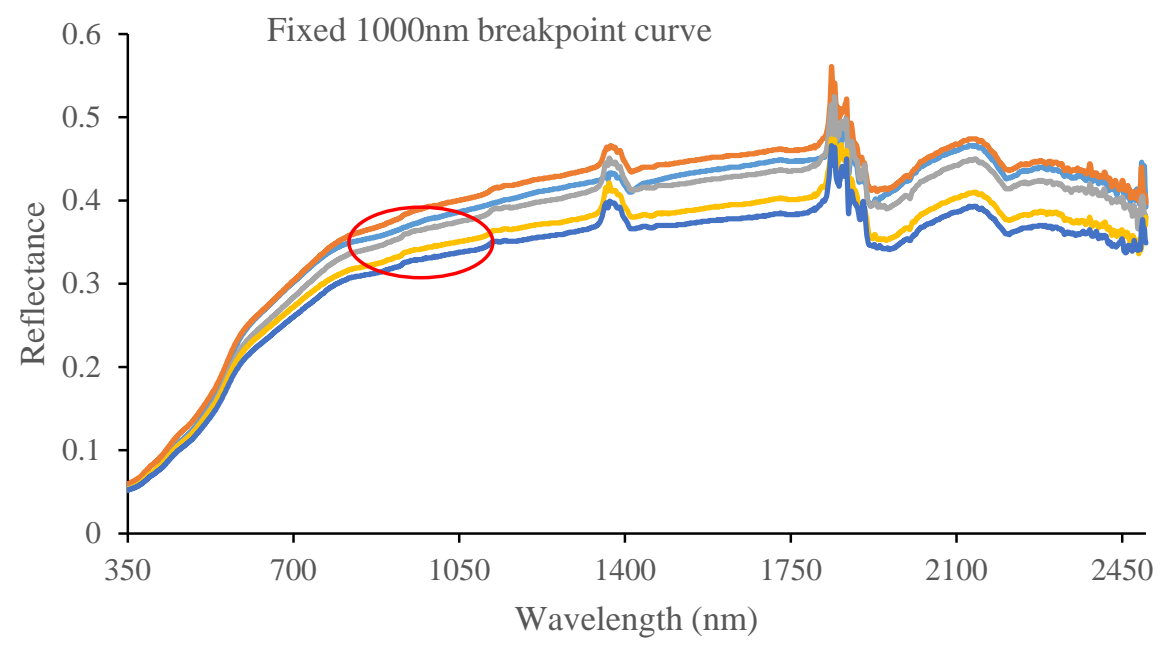

Figure 1. The repair of breakpoint $1000 \mathrm{~nm}$

\subsection{Differential transformation}

In addition to analysis of soil spectral, three transformations were made for finding the response regions of different heavy metal elements. First deviation and second deviation transformations can increase the correlation between reflectivity and heavy metal elements while eliminating or limiting the influence of partial linearity or near linear background.

$$
\begin{aligned}
& \rho^{\prime}\left(\lambda_{i}\right)=\left[\rho\left(\lambda_{i+1}\right)-\rho\left(\lambda_{i-1}\right)\right] / \Delta \lambda \\
& \rho^{\prime \prime}\left(\lambda_{i}\right)=\left[\rho^{\prime}\left(\lambda_{i+1}\right)-\rho^{\prime}\left(\lambda_{i-1}\right)\right] / \Delta \lambda
\end{aligned}
$$

The model results were verified by the decision factor R2 and the root mean square error RMSE.

$$
\begin{array}{r}
R^{2}=\frac{\sum_{i=1}^{n}\left(\hat{y}_{i}-\bar{y}_{i}\right)^{2}}{\sum_{i=1}^{n}\left(y_{i}-\bar{y}_{i}\right)^{2}} \\
R M S E=\sqrt{\frac{1}{n} \sum_{i=1}^{n}\left(\hat{y}_{i}-y_{i}\right)^{2}}
\end{array}
$$




\section{Results and Discussion}

The correlation coefficients of first deviation of Mn were 0.76 , respectively has reached a significant correlation. All the optimal PLSR models, the best modeling precision of the other eight heavy metals Mn, were 0.7852 (Table 2. Figure 2)

Table 2. The result of PLSR based on different pretreatment methods

\begin{tabular}{ccccccc}
\hline \multirow{2}{*}{ Elements } & \multirow{2}{*}{ Methods } & \multicolumn{3}{c}{ Calibration } & \multicolumn{2}{c}{ Validation } \\
\cline { 2 - 6 } & S+C & 0.7495 & 8.93 & 3 & 0.5553 & 10.9 \\
& C+FD & 0.7246 & 9.30 & 1 & 0.5545 & 10.8 \\
& C+SD & 0.694 & 9.67 & 1 & 0.8271 & 8.82 \\
& C+LOG & 0.7424 & 9.04 & 3 & 0.6493 & 9.74 \\
& NOR+S & 0.7325 & 12.4 & 3 & 0.7180 & 8.79 \\
& NOR+FD & 0.6033 & 8.35 & 1 & 0.6625 & 9.95 \\
& NOR+SD & 0.6844 & 9.56 & 1 & 0.5523 & 10.7 \\
& NOR+LOG & 0.7517 & 12.3 & 3 & 0.7255 & 9.02 \\
& MSC+S & 0.4021 & 8.60 & 1 & 0.8002 & 10.3 \\
& MSC+FD & 0.7852 & 9.19 & 2 & 0.8015 & 8.48 \\
& MSC+SD & 0.7057 & 9.32 & 1 & 0.8486 & 8.61 \\
& MSC+LOG & 0.4062 & 8.56 & 1 & 0.7835 & 10.2 \\
& SNV+S & 0.7707 & 9.18 & 3 & 0.6960 & 9.30 \\
& SNV+FD & 0.7324 & 10.8 & 1 & 0.5522 & 10.6 \\
& SNV+SD & 0.7233 & 9.84 & 1 & 0.8540 & 8.57 \\
& SNV+LOG & 0.7726 & 8.90 & 3 & 0.7159 & 9.12 \\
\hline
\end{tabular}

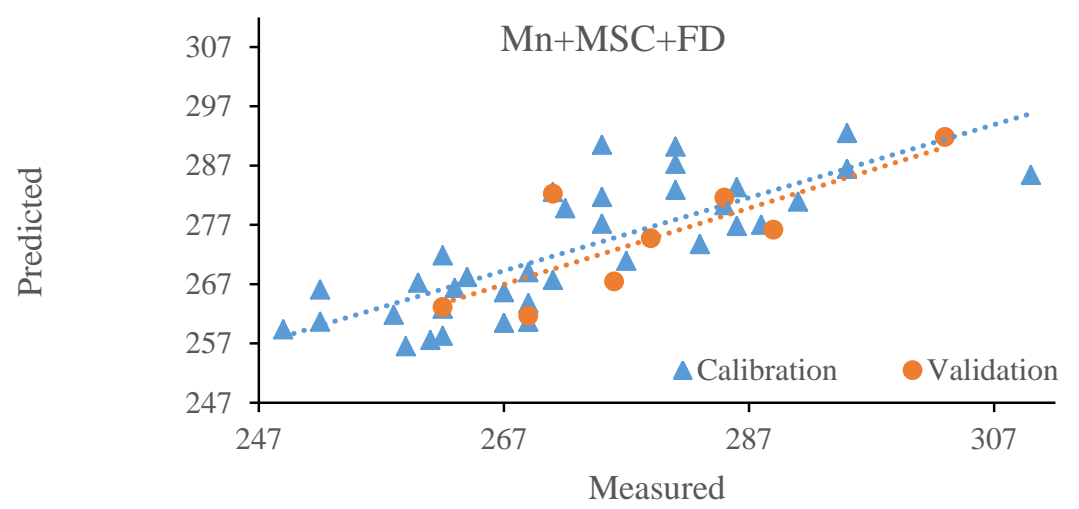

Figure 2. Comparison between observed and predicted based on different pretreatment

\section{Conclusion}

(1) The reflection spectra were processed by NOR, MSC and SNV respectively. The differential transformation can help to improve the correlation between the heavy metal elements and the reflection spectrum in the soil, and the use of the higher correlation band can significantly improve the stability and prediction ability of the model.

(2) The accuracy of Mn modeling was the highest, the prediction effect was better, $\mathrm{Rc}^{2}=0.7852$, $\mathrm{Rv}^{2}=0.8015$.

\section{Acknowledgments}

This work was financially supported by National Key Technology Support Program fund. We greatfully acknowledge the Institute of Land Engineering and Technology, Shanxi Provincial Land Engineering Construction Group, Xi'an, China. 


\section{References}

[1] Han, C.L, LIU, J.B, FU, X.M, et al. Hyperspectrum characteristics and Modeling of Organic Matter Content in Soils Developed from Trachytic Tephra, Chinese Journal of Soil Science, 2017, 47(1): 39 - 44.

[2] AL- ABBAS A H, SWAIN P H, BAUMGARDNER M F. Relating organic matter and clay content to the multispectral radiance of soils. Soil Science, 1972, 114(6): 477 - 485.

[3] LIU, H.Y, MAO, H.P, ZHU, W.J, et al. Rapid diagnosis of tomato N-P-K nutrition level based on hyperspectral technology. Chinese Society of Agricultural Engineering, 2015, 31: 212 - 220.

[4] LU, W.Y, LU, J.D, YAO, S.C, et al. Synchronization Detection of Nitrogen Phosphorus Potassium in Compound Fertilizer with Laser Induced Breakdown Spectroscopy. Chinese Journal of Lasers, 2015, 38(10): 10080031 - 6.

[5] KOOISTRA L, WEHRENS R, LEUVEN R S E W, et al. Possibilities of visible-near-infrared spectroscopy for the assessment of soil contamination in river floodplains. Analytica Chimica Acta, 2001, 446: 97 - 105.

[6] GRZEGORZ S, GREGORY W M, TOMASZ I S, et al. Near and mid-infrared diffuse reflectance spectroscopy for measuring soil metal content. Journal of Environment Quality, 2004, 33: 2056 - 2069.

[7] WU, D.W, WU, Y.Z, MA, H.R. Study on the Prediction of Soil Heavy Metal Elements Content Based on Mid-Infrared Diffuse Reflectance Spectra. Spectroscopy and Spectral Analysis, 2010, 30(6): 1498 - 1502. 